\title{
Erratum to: Genetic Population Structure of Thunnus albacares in the Central Pacific Ocean Based on mtDNA COI Gene Sequences
}

\author{
Weiwen $\mathrm{Li}^{1} \cdot$ Xinjun Chen ${ }^{1,2} \cdot$ Qianghua $\mathrm{Xu}^{1,2}$. \\ Jiangfeng $\mathrm{Zhu}^{1,2} \cdot$ Xiaojie Dai ${ }^{1,2} \cdot$ Liuxiong $\mathrm{Xu}^{1,2}$
}

\section{Erratum to: Biochem Genet (2015) 53:8-22 DOI 10.1007/s10528-015-9666-0}

The original version of this article does not contain any nucleotide sequence accession numbers.

The mtDNA COI sequences were deposited in GenBank under the following nucleotide sequence accession numbers:
KT211291， KT211292， KT211293， KT211294， KT211295， KT211296, KT211297, KT211298, KT211299, KT211300, KT211301, KT211302, KT211303, KT211304, KT211305, KT211306, KT211307, KT211308, KT211309, KT211310, KT211312, KT211313， KT211314, KT211315, KT211316, KT211317, KT211318, KT211319, KT211320, KT211321, KT211322, KT211323, KT211324, KT211325, KT211326, KT211327, KT211328, KT211329, KT211330, KT211331， KT211332， KT211333, KT211334, KT211335, KT211336, KT211337, KT211338, KT211339, KT211340, KT211341, KT211342, KT211343, KT211344, KT211345, KT211346, KT211347, KT211348, KT211349, KT211350, KT211351.

The online version of the original article can be found under doi:10.1007/s10528-015-9666-0.

Qianghua Xu

qhxu@shou.edu.cn

1 Key Laboratory of Sustainable Exploitation of Oceanic Fisheries Resources, Ministry of Education, College of Marine Sciences, Shanghai Ocean University, 999 Huchenghuan Road, Lingang New Harbor, Shanghai 201306, People's Republic of China

2 Collaborative Innovation Center for Distant-water Fisheries, Scientific Observing and Experimental Station of Oceanic Fishery Resources, Ministry of Agriculture, Shanghai Ocean University, Shanghai 201306, People's Republic of China 